\title{
Excesso de peso e ambiente de trabalho no setor público municipal
}

\author{
Overweight and workplace in \\ munucipal public sector
}

Patrícia Pinheiro de FREITAS ${ }^{1}$

Ada Ávila ASSUNÇÃO²

lara Barreto BASSI ${ }^{2}$

Aline Cristine Souza LOPES 3

\section{RE S U M O}

\section{Objetivo}

Identificar se características sociodemográficas, hábitos alimentares, situação de saúde e condições de trabalho estão relacionadas ao excesso de peso em servidores e empregados públicos municipais.

\section{Métodos}

Inquérito epidemiológico eletrônico (online) com servidores do município de Belo Horizonte, Minas Gerais, de setembro a dezembro de 2009. Investigaram-se informações sociodemográficas, de saúde, hábitos relacionados à alimentação e nutrição e informações sobre condições e ambiente de trabalho. Foi realizada análise de Regressão de Poisson $(p<0,05)$, ajustada por sexo, idade e tempo do funcionário na função.

\section{Resultados}

Um total de $44,4 \%$ dos participantes ( $n=4893$ ) apresentou sobrepeso, que prevaleceu em mulheres $(p<0,001)$ e pessoas com maior mediana de idade $(p<0,001)$, com filhos $(p<0,001)$, casadas ou em união estável $(p=0,002)$, e com menor nível educacional $(p<0,001)$ e status ocupacional $(p<0,001)$, determinados pelo International Socio-Economic Index. A regressão de Poisson encontrou associação entre sobrepeso e auto-relato de diabetes mellitus ( $R P=1,15$; IC95\%=1,03-1,27), hipercolesterolemia $(R P=1,24 ; I C 95 \%=1,15-1,32)$, hipertensão arterial $(\mathrm{RP}=1,47 ; \mathrm{IC} 95 \%=1,37-1,58)$, autoavaliação de saúde ( $\mathrm{RP}=1,20 ; \mathrm{IC} 95 \%=1,12-1,29)$, cozinhar habitualmente

\footnotetext{
${ }^{1}$ Universidade Federal de Minas Gerais, Escola de Enfermagem, Programa de Pós-Graduação em Enfermagem. Belo Horizonte, MG, Brasil.

2 Universidade Federal de Minas Gerais, Faculdade de Medicina, Núcleo de Estudos Saúde e Trabalho, Departamento de Medicina Preventiva e Social. Belo Horizonte, MG

3 Universidade Federal de Minas Gerais, Escola de Enfermagem, Departamento de Nutrição. Av. Alfredo Balena, 190, Santa Efigênia, 30130-100, Belo Horizonte, MG, Brasil. Correspondência para/Correspondence to: ACS LOPES. E-mail: $<$ alinelopesenf@gmail.com>.

Apoio: Fundação de Amparo à Pesquisa do Estado de Minas Gerais, PPM-00567-13.
} 
$(R P=1,11 ; \mid C 95 \% C l=1,04-1,20)$, consumir carne com gordura aparente $(R P=1,16$; IC95\%=1,08-1,24), e frequentemente não pausar durante o trabalho $(\mathrm{RP}=1,10 ; \mathrm{IC} 95 \%=1,03-1,18)$.

\section{Conclusão}

A relação entre ambiente de trabalho e excesso de peso, evidenciada pela associação com a privação de pausa no trabalho, denota a necessidade de medidas preventivas específicas que considerem as características do ambiente de trabalho.

Palavras-chave: Ambiente de trabalho. Condições de trabalho. Obesidade. Saúde do trabalhador. Sobrepeso.

\section{A B S T R A C T}

\section{Objective}

To determine whether sociodemographic characteristics, food habits, health status, and working conditions are related to excess weight in municipal civil servants.

\section{Methods}

This online epidemiological survey collected data about the sociodemographic characteristics, health, food habits, nutrition, and workplace of civil servants from the municipality of Belo Horizonte, Minas Gerais, from September to December 2009. Poisson regression adjusted for gender, age, and job duration was performed using a significance level of $5 \%(p<0.05)$.

\section{Results}

A total of $44.4 \%$ of the participants $(n=4,893)$ had excess weight, which prevailed in women $(p<0.001)$ and individuals with higher median age $(p<0.001)$, who had children $(p<0.001)$, who were married or had a partner $(p=0.002)$, and who had lower education level $(p<0.001)$ and occupational status, determined by the International Socioeconomic Index $(p<0.001)$. Poisson regression found associations between overweight and self-reported diabetes mellitus ( $P R=1.15 ; 95 \% C l=1.03-1.27)$, hypercholesterolemia $(P R=1.24 ; 95 \% C l=1.15-1.32)$, hypertension $(P R=1.47 ; 95 \% C l=1.37-1.58)$, health self-assessment $(P R=1.20 ; 95 \% C l=1.12-1.29)$, habitual cooking $(P R=1.11 ; 95 \% C l=1.04-1.20)$, eating meat with visible fat $(P R=1.16 ; 95 \% C l=1.08-1.24)$, and hardly taking work breaks (PR=1.10; C195\%: 1.03-1.18).

\section{Conclusion}

The relationship between workplace and excess weight, evidenced by its association with infrequent work breaks, shows the need of specific preventive measures that take into account workplace characteristics.

Keywords: Working environment. Working conditions. Obesity. Occupational health. Overweight.

\section{N T R O D U Ç Ã O}

O excesso de peso constitui um problema de saúde pública: a cada ano, 2,8 milhões de pessoas morrem por doenças a ele relacionadas', entre as quais figuram as patologias cardiovasculares, o diabetes Mellitus e o câncer ${ }^{2}$. No Brasil, essa condição atinge $52,5 \%$ da população ${ }^{3}$, sendo que as doenças crônicas associadas à obesidade representam $72,0 \%$ das causas de morte ${ }^{4}$.

Fatores genéticos e estilo de vida estão relacionados ao excesso de peso ${ }^{5}$. No que se refere à influência do ambiente, resultados recentes indicam sua relação tanto sobre as práticas alimentares quanto com a disposição com atividades físicas ${ }^{6}$. Ademais, evidencia-se, na literatura, a associação entre obesidade e ambientes de trabalho com características hostis e longas jornadas 7,8 .

Indivíduos com excesso de peso são mais susceptíveis ao absenteísmo-doença, gerando prejuízos para a produção ${ }^{8}$. Essas evidências e hipóteses geram preocupação não apenas com relação às perdas no trabalho, mas com a saúde 
dos trabalhadores. Esse fato tem justificado o interesse dos pesquisadores em investigar a influência das condições de trabalho sobre o estado nutricional $^{5,9,10}$.

Luckhaupt et al. ${ }^{7}$, em um inquérito de saúde, detectaram maior prevalência de obesidade (36,3\%) em funcionários da administração pública nos Estados Unidos da América, quando comparada à prevalência na população total de trabalhadores estudados $(27,7 \%)$ naquele país e na população geral $(32,6 \%)^{1}$. Nessa direção, este artigo objetivou identificar se características sociodemográficas, hábitos alimentares, situação de saúde e condições de trabalho estavam relacionadas ao excesso de peso em um grupo de servidores e empregados públicos municipais.

\section{M É T O D O S}

Realizou-se inquérito epidemiológico por questionário eletrônico respondido por servidores (ocupantes de cargo efetivo por regime estatutário) e empregados (contratados pelo município sob regime de Consolidação das Leis do Trabalho) do município, de setembro a dezembro de 2009. O município em questão se encontra no estado de Minas Gerais e possui população estimada de 2412937 habitantes.

A divulgação da pesquisa ocorreu com o auxílio do corpo gestor, em todos os órgãos da Prefeitura do município, mediante chamadas na Internet e Intranet, mensagens em contracheques e sensibilização junto aos sindicatos representativos dos servidores e empregados municipais para a importância da participação na pesquisa. Semanalmente, estratégia de divulgação foi empregada em locais com menor adesão. Todos os 38304 servidores e empregados públicos do município foram convidados a participar, sendo que, destes, 5646 (14,7\%) responderam ao questionário disponibilizado em sítio da Internet.

A pesquisa abordou questões sociodemográficas (idade, sexo, número de filhos, escolaridade, ocupação, estado civil e renda), de saúde (morbidades referidas: hipertensão arterial, hipercolesterolemia, diabetes Mellitus, gastrite, ansiedade/depressão e distúrbios do sono; tabagismo; autoavaliação de saúde e qualidade de vida; incapacidade de realização de atividades habituais por motivo de saúde; frequência de prática de atividade física; peso e altura referidos), hábitos relacionados à alimentação e nutrição (hábito de cozinhar e fazer compras de alimentos; hábito de pesar-se; consumo de frutas e hortaliças, consumo de carne com gordura e pele de frango; hábito de adicionar sal na comida pronta, exceto salada) e informações sobre condições e ambiente de trabalho (licença médica recente, doenças relacionadas ao trabalho, jornada, ficar em pé ou sentado no trabalho, ficar sem fazer pausa, tempo disponível para alimentar-se, lanche oferecido pelo empregador, existência de sala de descanso e copa/refeitório, percepção à exigência no trabalho).

Para definição de status ocupacional, foi construído o International Socio-Economic Index (ISEI) com as informações sobre ocupação, escolaridade e renda. A escala foi obtida com base na Classificação Brasileira de Ocupações e, quando não encontrada correspondência com o cargo foi utilizado o código que mais se assemelhasse à função com base em: atribuição e competência, escolaridade mínima exigida, salário base inicial e local de trabalho. Uma vez que a sintaxe disponibilizada pela International Stratification and Mobility File se baseia nos códigos da CIUO88 para a obtenção da escala ISEI, a correspondência da Prefeitura de Belo Horizonte/CBO foi convertida conforme tabela do Ministério do Trabalho e Emprego.

Para avaliar o excesso de peso, variável desfecho, foram utilizadas as medidas de peso e altura autorreferidas. Cabe destacar a relevância do uso do Índice de Massa Corporal (IMC) relatado, uma vez comprovada sua boa confiabilidade e precisão em outros inquéritos ${ }^{11}$. Assim, neste estudo, o estado nutricional foi verificado pelo IMC, classificado segundo as recomendações da World Health Organization ${ }^{12}$. Para análise de dados, os participantes foram agrupados de acor- 
do com o diagnóstico de excesso de peso (IMC $\geq 25 \mathrm{~kg} / \mathrm{m}^{2}$ ).

Realizou-se análise descritiva das informações obtidas. Para verificar a distribuição das variáveis contínuas, utilizou-se o teste estatístico de Kolmogorov-Smirnov. Devido ao seu caráter assimétrico, as variáveis testadas foram apresentadas em mediana e intervalo interquartílico $\left(\mathrm{P}_{25}\right.$; $\left.\mathrm{P}_{75}\right)$.

Foram utilizados os testes estatísticos Qui-quadrado e Mann Whitney para comparação do desfecho com as variáveis categóricas e numéricas, respectivamente. Considerou-se um nível de significância menor que 0,05 em todos os testes.
Para identificar os fatores associados à variável desfecho, excesso de peso, utilizou-se a análise de Regressão de Poisson com variância robusta. Foram criados quatro blocos com base nas características das variáveis: 1) informações sociodemográficas; 2) de saúde; 3) hábitos de vida, 4) condições e ambiente de trabalho. As covariáveis cuja associação com o desfecho foi constatada na análise bivariada com significância menor que 0,20 foram incluídas pelo método backward no modelo de Regressão de Poisson, permanecendo aquelas com nível de significância menor que $5 \%$ e sendo excluídas as com relação de multicolinearidade. Após essas análises, foi

Tabela 1. Características sociodemográficas e de saúde de servidores e empregados públicos segundo a presença de excesso de peso. Belo Horizonte (MG), 2009.

\begin{tabular}{|c|c|c|c|c|c|}
\hline \multirow{2}{*}{ Variáveis } & \multirow{2}{*}{$\mathrm{N}$} & \multirow{2}{*}{ Total } & \multicolumn{2}{|c|}{ Excesso de peso } & \multirow{2}{*}{ Valor $p$} \\
\hline & & & Não (n=2 719) & $\operatorname{sim}(n=2174)$ & \\
\hline Idade (anos) & 4851 & $42,0(33-49)$ & $40,0(31-47)$ & $44,0(20-71)$ & $<0,001^{a}$ \\
\hline Sexo & & & & & $<0,001^{\text {b }}$ \\
\hline Feminino & 4888 & 66,3 & 71,6 & 59,7 & \\
\hline Tem filhos & 4889 & 63,0 & 58,3 & 68,8 & $<0,001^{b}$ \\
\hline Escolaridade & 4886 & & & & $<0,001^{b}$ \\
\hline Até 4 anos & & 3,3 & 2,7 & 3,9 & \\
\hline De 5 a 8 anos & & 4,7 & 3,7 & 5,8 & \\
\hline De 9 a 11 anos & & 24,4 & 22,4 & 26,8 & \\
\hline 12 ou mais & & 67,6 & 71,2 & 63,5 & \\
\hline International Socio-Economic Index. & 4655 & $43,0(29,0-69,0)$ & $50,0(29,0-69,0)$ & $38,0(23,0-88,0)$ & $<0,001^{a}$ \\
\hline Estado civil & 4886 & & & & $0,002^{b}$ \\
\hline Sem companheiro & & 43,4 & 45,4 & 41,0 & \\
\hline Com companheiro & & 56,6 & 54,0 & 59,0 & \\
\hline \multicolumn{6}{|l|}{ Morbidades referidas } \\
\hline Hipertensão arterial & 4668 & 19,1 & 10,1 & 30,4 & $<0,001^{b}$ \\
\hline Hipercolesterolemia & 4668 & 19,8 & 14,0 & 27,0 & $<0,001^{\text {b }}$ \\
\hline Diabetes Mellitus & 4667 & 3,9 & 2,0 & 6,4 & $<0,001^{b}$ \\
\hline Gastrite & 4669 & 24,0 & 22,8 & 25,4 & $0,043^{b}$ \\
\hline Ansiedade/depressão & 4657 & 22,7 & 21,0 & 24,8 & $0,002^{b}$ \\
\hline Distúrbios do sono & 4664 & 25,1 & 23,0 & 27,8 & $<0,001^{b}$ \\
\hline Tabagismo & 4731 & 10,5 & 10,2 & 10,9 & $0,423^{b}$ \\
\hline $\begin{array}{l}\text { Deixou de fazer atividade habitual por } \\
\text { motivo de saúde - últimos } 3 \text { meses }\end{array}$ & 4572 & 22,5 & 21,8 & 23,5 & $0,183^{b}$ \\
\hline Saúde boa/muito boa & 4670 & 79,3 & 84,7 & 72,4 & $<0,001^{b}$ \\
\hline Qualidade de vida boa/muito boa & 4440 & 75,0 & 76,8 & 72,7 & $0,002^{a}$ \\
\hline Atividade física - últimos 3 meses & 2476 & & & & $0,600^{b}$ \\
\hline Menos de 3 vezes na semana & & 52,5 & 53,0 & 51,9 & \\
\hline De 3 a 7 vezes por semana & & 47,5 & 47,0 & 48,1 & \\
\hline
\end{tabular}

Nota: ${ }^{\text {T} T e s t e ~ d e ~ M a n n ~ W h i t n e y, ~}{ }^{\mathbf{b}}$ Teste de Qui-quadrado. 
construído um modelo final repetindo os procedimentos anteriores, ajustado pelas variáveis sexo, idade e tempo do funcionário na função. Utilizou-se, como medida de efeito, a Razão de Prevalência (RP) com Intervalo de Confiança de 95\% (IC95\%).

As análises estatísticas foram realizadas com o auxílio do software Stata (Stata Corporation, College Station, Texas, Estados Unidos da América) versão 11.0 e Statistical Package for the Social Sciences (SPSS Inc., Chicago, Illinóis, Estados Unidos da América) versão 17.0. Quanto aos aspectos éticos, este estudo foi aprovado pelo Comitê de Ética em Pesquisa da Prefeitura Municipal de Belo Horizonte (Parecer $n^{\circ}$ 0054.0.410.000.09a). O participante era informado sobre o estudo e o acesso ao questionário somente era realizado após o aceite do Termo de Consentimento Livre e Esclarecido.

\section{RES U L T A D O S}

Do total de 5646 servidores e funcionários públicos respondentes, foram excluídos aqueles que não informaram o peso e/ou altura $(n=694)$ e as gestantes $(n=59)$, totalizando 4893 participantes analisados. Estes eram, em sua maioria, mulheres $(66,3 \%)$ com mediana de idade de 42 anos e escolaridade superior a 11 anos.
A prevalência do excesso de peso foi de $44,4 \%$, variando de acordo com as características sociodemográficas, de saúde $(p<0,05)$ e status ocupacional, mensurado pelo ISEI $(p<0,001)$ (Tabela 1). Indivíduos com excesso de peso também apresentaram maior proporção do hábito de cozinhar $(p=0,001)$, ir ao supermercado ou feira $(p=0,005)$, consumir carne com gordura aparente $(p<0,001)$ e pele de frango $(p=0,032)$ (Tabela 2$)$. Já a análise das condições e ambiente de trabalho mostrou a relação do excesso de peso com a jornada de trabalho $(p=0,018)$ e atividades onde o profissional necessita permanecer em pé por muito tempo $(p=0,004)$ (Tabela 3$)$.

Na análise multivariada de Poisson, após ajuste pelas variáveis sexo e idade, o excesso de peso dos trabalhadores municipais foi associado a: relatar o diagnóstico de diabetes Mellitus $(R P=1,15 ;$ IC95\%=1,03-1,27), hipercolesterolemia $(R P=1,24 ; \quad I C 95 \%=1,15-1,32)$ e hipertensão arterial ( $R P=1,47$; IC $95 \%=1,37-1,58)$; não considerar a saúde como boa/muito boa ( $\mathrm{RP}=1,20$; IC95\%=1,12-1,29); ter hábito de cozinhar $(\mathrm{RP}=1,11$; IC95\%=1,04-1,20); consumir carne com gordura aparente $(R P=1,16$; IC $95 \%=1,08-1,24)$; e não realizar pausa durante o trabalho na maior parte do tempo $(R P=1,10 ; \mathrm{IC} 95 \%=1,03-1,18)$ (Tabela 4).

Tabela 2. Aspectos alimentares e nutricionais de servidores e empregados públicos segundo a presença de excesso de peso. Belo Horizonte (MG), 2009.

\begin{tabular}{|c|c|c|c|c|c|}
\hline \multirow{2}{*}{ Variáveis } & \multirow{2}{*}{$\mathrm{N}$} & \multirow{2}{*}{ Total (\%) } & \multicolumn{2}{|c|}{ Excesso de peso } & \multirow{2}{*}{ Valor $p^{*}$} \\
\hline & & & Não $(n=2719)$ & $\operatorname{Sim}(n=2174)$ & \\
\hline Há quanto tempo você se pesou pela última vez & 4886 & & & & 0,207 \\
\hline Menos de 1 mês & & 59,1 & 58,2 & 60,2 & \\
\hline Mais de 1 mês & & 37,2 & 37,8 & 36,6 & \\
\hline Não lembro & & 3,7 & 4,0 & 3,2 & \\
\hline Hábito de cozinhar & 4748 & 64,5 & 62,5 & 67,0 & 0,001 \\
\hline Hábito de comprar alimentos & 4749 & 88,3 & 87,1 & 89,7 & 0,005 \\
\hline $\begin{array}{l}\text { Consumo regular de frutas e hortaliças - } 6 \text { vezes } \\
\text { na semana ou mais }\end{array}$ & 4743 & 48,5 & 49,6 & 47,1 & 0,098 \\
\hline Consumo de carne com gordura & 4603 & 25,3 & 21,6 & 29,8 & 0,000 \\
\hline Consumo de pele de frango & 4637 & 24,9 & 23,7 & 26,4 & 0,032 \\
\hline Adiciona sal na comida pronta & 4749 & 17,1 & 17,5 & 16,7 & 0,460 \\
\hline
\end{tabular}

Nota: *Teste de Qui-quadrado. 
Tabela 3. Condições e ambiente de trabalho de servidores e empregados públicos segundo a presença de excesso de peso. Belo Horizonte (MG), 2009.

\begin{tabular}{|c|c|c|c|c|c|}
\hline \multirow{2}{*}{ Variáveis } & \multirow{2}{*}{ N } & \multirow{2}{*}{ Total } & \multicolumn{2}{|c|}{ Excesso de peso } & \multirow{2}{*}{ Valor $p$} \\
\hline & & & Não (n=2 719) & $\operatorname{Sim}(n=2174)$ & \\
\hline Licença médica nos últimos 12 meses & 4651 & 44,7 & 44,1 & 45,6 & $0,295^{a}$ \\
\hline Tem/teve doença relacionada ao trabalho & 4642 & 32,8 & 31,7 & 34,2 & $0,056^{\mathrm{a}}$ \\
\hline Jornada trabalho (minutos/dia) & 4540 & $480(480-500)$ & $480(480-480)$ & $480(480-540)$ & $0,018^{\mathbf{b}}$ \\
\hline Ficar em pé no trabalho - às vezes ou sempre & 4534 & 56,6 & 54,7 & 59,0 & $0,004^{a}$ \\
\hline Ficar sentado no trabalho - às vezes ou sempre & 4531 & 70,9 & 71,2 & 70,5 & $0,608^{a}$ \\
\hline Não faz pausa no trabalho - às vezes ou sempre & 4519 & 53,4 & 52,2 & 55,0 & $0,059^{a}$ \\
\hline Tem tempo para alimentar-se no trabalho & 4514 & 86,2 & 86,2 & 86,0 & $0,852^{a}$ \\
\hline Empregador oferece lanche & 4488 & 55,4 & 54,8 & 56,2 & $0,342^{a}$ \\
\hline Tem copa/refeitório no trabalho & 4517 & 74,6 & 74,8 & 74,3 & $0,699^{a}$ \\
\hline Trabalho tem sala de descanso & 4520 & 12,4 & 12,3 & 12,6 & $0,736^{\mathrm{a}}$ \\
\hline Trabalho exigente - às vezes ou sempre & 4464 & 80,2 & 79,6 & 80,8 & $0,320^{a}$ \\
\hline
\end{tabular}

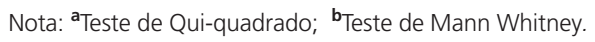

Tabela 4. Modelo multivariado de variáveis associadas ao excesso de peso. Belo Horizonte (MG), 2009.

\begin{tabular}{|c|c|c|}
\hline Variáveis & Excesso de peso RP (IC95\%) & Valor $p^{*}$ \\
\hline \multicolumn{3}{|l|}{ Diabetes Mellitus } \\
\hline Não & 1,0 & \multirow{2}{*}{0,011} \\
\hline $\operatorname{sim}$ & $1,15(1,03 ; 1,27)$ & \\
\hline \multicolumn{3}{|l|}{ Hipercolesterolemia } \\
\hline Não & 1,0 & \multirow{2}{*}{$<0,001$} \\
\hline Sim & $1,24(1,15 ; 1,32)$ & \\
\hline \multicolumn{3}{|l|}{ Hipertensão arterial } \\
\hline Não & 1,0 & \multirow{2}{*}{$<0,001$} \\
\hline $\operatorname{Sim}$ & $1,47(1,37 ; 1,58)$ & \\
\hline \multicolumn{3}{|c|}{ Considera saúde boa/muito boa } \\
\hline $\operatorname{sim}$ & 1,0 & \multirow{2}{*}{$<0,001$} \\
\hline Não & $1,20(1,12 ; 1,29)$ & \\
\hline \multicolumn{3}{|c|}{ Hábito de cozinhar (\%) } \\
\hline Não & 1,0 & \multirow{2}{*}{0,001} \\
\hline Sim & $1,11(1,04 ; 1,20)$ & \\
\hline \multicolumn{3}{|c|}{ Comer carne com gordura aparente } \\
\hline Não & 1,0 & \multirow{2}{*}{$<0,001$} \\
\hline Sim & $1,16(1,08 ; 1,24)$ & \\
\hline \multicolumn{3}{|c|}{ Não faz pausa durante o trabalho } \\
\hline Nunca/raramente & 1,0 & \multirow{2}{*}{0,003} \\
\hline Às vezes/sempre & $1,10(1,03 ; 1,18)$ & \\
\hline
\end{tabular}

Nota: "Regressão de Poisson (RP); Modelo ajustado por sexo, idade, tempo do funcionário na função; Ajuste do modelo: Good of fitness = 1,00.

\section{I S C U S S Ã O}

A prevalência de excesso de peso no grupo dos servidores e empregados municipais foi inferior à população brasileira ${ }^{3}$, estando associada não somente às condições de saúde e alimentação, mas também à privação de pausa durante a jornada de trabalho. Esses resultados indicam a influência do contexto de trabalho.

Valor semelhante de excesso de peso $(45,0 \%)$ foi encontrado em estudo realizado com 
servidores públicos federais no Brasil ${ }^{8}$. Contudo, prevalências superiores, 53,0\%, 53,6\%, 61,9\% e $71,5 \%$, foram encontradas, respectivamente, em servidores públicos ${ }^{13}$, em uma amostra de trabalhadores da indústria metal-mecânica ${ }^{14}$, em trabalhadores de um Centro de Saúde-Escola ${ }^{15}$ e em trabalhadores de uma cozinha hospitalar ${ }^{16}$.

Servidores e empregados municipais que laboram sob privação de pausas apresentaram maior prevalência de excesso de peso quando comparados ao grupo que informou usufruir dessas pausas. Esse resultado é inédito no Brasil, onde há carência de estudos nessa direção. Ressalta-se que, apesar da jornada de trabalho ter sido relacionada ao excesso de peso na análise bivariada (com medianas de tempo de trabalho semelhantes e intervalo de confiança superior para o grupo com excesso de peso), a variável não permaneceu no modelo final ajustado. Entretanto, a falta de autonomia na execução das tarefas profissionais e a menor satisfação com as atividades desen-volvidas, jornada prolongada e trabalho em turnos já teve sua relação demonstrada com o ganho ponderal5, $5,10,13$. Assim, em situação de jornada de trabalho prolongado, as pausas são fundamentais para permitir a recuperação da capacidade física e mental. Sob privação do tempo para recuperação é possível que o indivíduo ultrapasse seus limites fisiológicos, gerando respostas negativas, como a ansiedade, a qual pode perturbar a duração e a qualidade do sono, alterar a secreção de hormônios fundamentais para o metabolismo sadio e tornar o indivíduo mais propenso a buscar alívio imediato das tensões por meio de comportamentos prejudiciais à saúde, como compulsão para comer e tabagismo ${ }^{5,17}$.

A associação com morbidades e pior avaliação de saúde era esperada, corroborando o impacto negativo das alterações do peso sobre a saúde ${ }^{10,13}$. O relato de alterações nos níveis de colesterol sanguíneo apresentado é consistente com a literatura $8,18,19$. Concomitantemente, nota-se o predomínio de respondentes que relataram consumo de carne com gordura aparente, justi- ficando a elaboração de medidas de prevenção e promoção da saúde, tendo em vista a relação do excesso de peso e da hipercolesterolemia com essa prática alimentar inadequada.

Quanto aos hábitos, a prática de cozinhar foi diretamente relacionada ao peso corporal excessivo. Esse resultado foi inesperado, pois a prática implicaria em restrições ao consumo de alimentos prontos ou pré-fabricados, os quais favorecem a ingestão de açúcar, gordura e sódio. Vale mencionar, contudo, a possibilidade de causa-lidade reversa, dado o desenho transversal do estudo. O hábito de cozinhar, nesse caso, pode ser uma tentativa daqueles com comprovado excesso de peso de adotarem hábitos alimentares mais saudáveis.

As características ocupacionais dos participantes possivelmente produziram resultados menos desfavoráveis quando comparados aos estudos os quais focalizaram outras categorias ocupacionais, por se tratar de uma amostra empregada e protegida quanto aos direitos sociais. Focalizando a situação no mercado de trabalho, pesquisas verificaram que estar inserido no trabalho sem proteção social e estar desempregado associava-se, por exemplo, à maior prevalência de tabagismo entre homens e mulheres, independentemente da escolaridade, da renda e da presença ou não de problemas de saúde diretamente relacionados a esse hábito ${ }^{17}$.

Este estudo utilizou como desfecho o excesso de peso, obtido a partir de medidas referidas. A utilização de medidas de peso e estatura autorreferidas é uma estratégia comum utilizada em inquéritos que possibilitam o desenvolvimento de pesquisas com grandes amostras em menor tempo e com baixo custo ${ }^{3}$. A pequena diferença nas estimativas com o uso de medidas referidas não parece produzir distorções estimativas globais ou estudos de associação entre valores antropométricos e fatores de risco à saúde ${ }^{11}$. Portanto, acredita-se que esse aspecto não constitui uma limitação metodológica possível de comprometer qualidade. 
Devido ao tipo de amostra, comparações com outras populações merecem cautela. A taxa de resposta do inquérito foi de $14 \%$, apesar das estratégias para estimular a adesão dos elegíveis e diminuir as disparidades no acesso ao veículo da pesquisa (foram disponibilizados laptops em áreas onde não havia esses equipamentos). Apesar do limite da superestimação dos resultados devido à baixa adesão ao estudo, provavelmente o prejuízo é de outra ordem: subestimação dos resultados, porque é evidente a super-representação dos sujeitos com maior escolaridade e status ocupacional superior ${ }^{20}$, características as quais refletem em melhor condição de saúde do referido grupo.

O presente estudo ganha em relevância tendo em vista o número de participantes (superior a 5 mil servidores), a incorporação de diferentes ocupações e a variedade de medidas referentes às características dos indivíduos e do trabalho. A estabilidade de emprego da população foco abre possibilidade de monitoramento permanente de possíveis disparidades e avaliação de programas sob a ótica da equidade, essenciais para que esses trabalhadores alcancem e conservem seu potencial de saúde.

Cabe destacar as potencialidades conferidas pela estratégia de coleta de dados via Web. Essa estratégia tem sido considerada mais vantajosa do que aquelas realizadas por meio dos veículos tradicionais devido ao custo reduzido, à facilidade e rapidez na administração, à maior garantia de anonimato, ao alcance em larga escala e ao tempo curto para resposta. Alguns autores sugerem que as pesquisas online são apropriadas por alcançarem respostas completas e por não se configurarem como respostas apresentadas para "agradar" o entrevistador ${ }^{21,22}$. Não obstante, riscos são possíveis, como a impossibilidade de controlar as amostras e de identificar a sua representatividade por causa da garantia do anonimato. Devido a esse último motivo, não foi controlado o local ou o computador de onde o questionário foi acessado ${ }^{23}$.

Os resultados obtidos reforçam a necessidade de medidas preventivas específicas aos fatores de risco modificáveis identificados, com destaque para a privação de pausas no trabalho. Ademais, o ambiente de trabalho é considerado espaço propício para a realização de atividades de educação em saúde. Sendo assim, são desejáveis novas abordagens para grupos ocupacionais específicos, visando contribuir para identificar vulnerabilidades ocupacionais e desenvolver políticas pró-equidade e monitoramento das ações propostas.

\section{OLABORADORES}

PP FREITAS contribuiu substancialmente na concepção, planejamento, análise e interpretação dos dados e elaboração do manuscrito. AA ASSUNÇÃO realizou delineamento do projeto e captação de recursos; contribuiu na concepção, planejamento, bem como na revisão crítica do conteúdo. IB BASSI e ACS LOPES contribuíram na concepção, planejamento e revisão crítica do conteúdo.

\section{REFERÊ NCIAS}

1. World Health Organization. WHO fact files: Ten facts on obesity. Geneva: Who; 2014 [cited 2014 Jan 31]. Available from: http://www.who.int/ features/factfiles/obesity/en/ index.html

2. Silventoinen $\mathrm{K}$, Tatsuse $\mathrm{T}$, Martikainen $\mathrm{P}$, Rahkonen $O$, Lahelma E, Sekine M, et al. Occupational class differences in body mass index and weight gain in Japan and Finland. J Epidemiol. 2013; 23(6):443-50. http://dx.doi.org/10.2188/jea.JE20130023

3. Brasil. Ministério da Saúde. Secretaria de Vigilância em Saúde. Vigitel Brasil 2014: vigilância de fatores de risco e proteção para doenças crônicas por inquérito telefônico. Brasília: Ministério da Saúde; 2015.

4. Schimidt MI, Duncan BB, Silva GA, Menezes AM, Monteiro CA, Barreto SM, et al. Chronic noncommunicable diseases in Brazil: Burden and current challenges. Lancet. 2011; 377(9781):1949-61. http://dx.doi.org/10.1016/\$0140-6736(11)60135-9

5. Solovieva S, Lallukka T, Virtanen M, Viikari-Juntura E. Psychosocial factors at work, long work hours, and obesity: A systematic review. Scand J Work Environ Health. 2013; 39(3):241-58. http://dx. doi.org/10.5271/sjweh.3364 
6. Lewin A, Pannier B, Méline J, Karusisi N, Thomas F. Residential neighborhood, geographic work environment, and work economic sector: Associations with body fat measured by bioelectrical impedance in the RECORD Study. Ann Epidemiol. 2014; 24(3):180-6. http://dx.doi.org/ 10.1016/j.annepidem.2013.12.001

7. Luckhaupt SE, Cohen MA, Li J, Calvert GM. Prevalence of obesity among U.S. workers and associations with occupational factors. Am J Prev Med. 2014; 46(3):237-48. http://dx.doi.org/10.10 16/j.amepre.2013.11.002

8. Oliveira RAR, Moreira OC, Lopes PRNR, Amorim W, Breguez MS, Marins JCB. Variáveis bioquímicas, antropométricas e pressóricas como indicadores de risco cardiovascular em servidores públicos. Fisioter Mov. 2013; 26(2):369-77. http://dx.doi.org/10.15 90/S0103-51502013000200014

9. Blanch A, Aluja A. Psychosocial work dimensions, personality, and body mass index: Sex differences. Int J Occup Med Environ Health. 2013; 26(4):572-80. http://dx.doi.org/10.2478/s13382-013-0121-3

10. Kim MJ, Son KH, Park HY, Choi DJ, Yoon CH, Lee $\mathrm{HY}$, et al. Association between shift work and obesity among female nurses: Korean Nurses' Survey. BMC Public Health. 2013; 13:1204. http:// dx.doi.org/10.1186/1471-2458-13-1204

11. Conde WL, Oliveira DR, Borges CA, Baraldi LG. Consistência entre medidas antropométricas em inquéritos nacionais. Rev Saúde Pública. 2013; 47(1):69-76. http://dx.doi.org/10.1590/S0034-8 9102013000100010

12. World Health Organization. Physical status: The use and Interpretation of Anthropometry. Technical Report Series, n 854. Geneva: WHO; 1995.

13. Höfelmann DA, Blank N. Auto-avaliação de saúde entre trabalhadores de uma indústria no Sul do Brasil. Rev Saúde Pública. 2007; 41(5):777-87. http://dx.doi.org/10.1590/S0034-89102007000 500012

14. Fonseca MJM, Faerstein E, Chor D, Lopes CS, Andreozzi VL. Associações entre escolaridade, renda e Índice de Massa Corporal em funcionários de uma universidade no Rio de Janeiro, Brasil: Estudo Pró-Saúde. Cad Saúde Pública. 2006; 22(11):2359-67. http://dx.doi.org/10.1590/S0102-311X2006001 100010
15. Nascimento LC, Mendes IJM. Perfil de saúde dos trabalhadores de um Centro de Saúde-Escola. Rev Latino-Am Enfermagem. 2002; 10(4):502-8. http:// dx.doi.org/10.1590/S0104-11692002000400006.

16. Boclin KLS, Blank N. Excesso de peso: característica dos trabalhadores de cozinhas coletivas? Rev Bras Saúde Ocup. 2006; 31(113):41-7. http://dx.doi.org/ 10.1590/S0303-76572006000100005

17. Giatti L, Barreto SM. Tabagismo, situação no mercado de trabalho e gênero: análise da PNAD 2008. Cad Saúde Pública. 2011; 27(6):1132-42. http:// dx.doi.org/10.1590/S0102-311X2011000600010

18. Cassani RSL, Nobre F, Filho Pazin A, Schmidth A. Prevalência de fatores de risco cardiovascular em trabalhadores de uma indústria brasileira. Arq Bras Cardiol. 2009; 92(1):16-22. http://dx.doi.org/10.15 90/S0066-782X2009000100004

19. Barel M, Louzada GCA, Monteiro HL, Amaral SL. Associação dos fatores de risco para doenças cardiovasculares e qualidade de vida entre servidores da saúde. Rev Bras Educ Fís Esporte. 2010; 24(2):293-303. http://dx.doi.org/10.1590/\$1807-55 092010000200012

20. Rodrigues CS, Freitas RM, Assunção AÁ, Bassi IB, Medeiros AM. Absenteísmo-doença segundo autorrelato de servidores públicos municipais em Belo Horizonte. R Bras Est Pop. 2013; 30(Supl.):S135-54. http://dx.doi.org/1590/S0102-3098201300040 0009

21. Evans JR, Mathur A. The value of online surveys. Internet Res. 2005; 15:195-219. http://dx.doi.org/10. 1108/10662240510590360

22. Ganassali S. The Influence of the Design of Web Survey Questionnaires on the Quality of Responses. Surv Res Methods. 2008; 2(1):21-32. http://dx.doi. org/10.18148/srm/2008.v2i1.598

23. Fox J, Murray C, Warm A. Conducting research using web-based questionnaires: Practical, methodological, and ethical considerations. Int J Soc Res Methodol. 2003; 6(20):167-80. http://dx. doi.org/10.1080/13645570210142883

Recebido: dezembro 15, 2014 Versão final: março 2, 2016 Aprovado: abril 12, 2016 
